Pacific Journal of Mathematics

DIFFERENTIABLE MAPS WITH O-DIMENSIONAL CRITICAL
SET. I 


\title{
DIFFERENTIABLE MAPS WITH 0-DIMENSIONAL CRITICAL SET, I
}

\author{
P. T. Church and J. G. TImourian
}

Let $f: M^{n} \rightarrow N^{p}$ be $C^{n}$ with $n-p=0$ or 1 , let $p \geqq 2$, and let $R_{p-1}(f)$ be the critical set of $f$. If $\operatorname{dim}\left(R_{p-1}(f)\right) \leqq 0$ and $\operatorname{dim}\left(f\left(R_{p-1}(f)\right) \leqq p-2\right.$, then (1.1) at each $x \in M^{n}, f$ is locally topologically equivalent to one of the following maps:

(a) the projection map $\rho: R^{n} \rightarrow R^{p}$,

(b) $\sigma: C \rightarrow C$ defined by $\sigma(z)=z^{d}(d=2,3, \ldots)$, where $C$ is the complex plane, or

(c) $\tau: C \times C \rightarrow C \times R$ defined by $\tau(z, w)=\left(2 z \cdot \bar{w},|w|^{2}-|z|^{2}\right)$, where $\bar{w}$ is the complex conjugate of $w$.

In particular, either $f$ is locally topologically equivalent to $\rho$ at each $x \in M^{n}$, or $(n, p)=(2,2)$ or $(4,3)$.

In a sequel the hypothesis on $\operatorname{dim} f\left(R_{p-1}(f)\right)$ is eliminated.

For a $C^{r}(r \geqq 1)$ map $f: M^{n} \rightarrow N^{p}$ let $R_{q}(f)$ be the set of points $x \in M^{n}$ at which the rank of (the derivative map of) $f$ is at most $q$. The critical set of $f$ is defined to be $R_{p-1}(f)$ (in case $n<p, R_{p-1}(f)=$ $\left.M^{n}\right)$, and according to the Rank Theorem [2, p. 155] at each $x \in M^{n}-$ $R_{p-1}(f), f$ is locally $C^{r}$ equivalent (1.3) to the map $\rho: R^{n} \rightarrow R^{p}$ defined by $\rho\left(x_{1}, x_{2}, \cdots x_{n}\right)=\left(x_{1}, x_{2}, \cdots, x_{p}\right)$. Thus (1.1) is a generalization of the Rank Theorem for $n-p=0$ or 1 , and $p \geqq 2$; moreover for $n-p=$ 1 it answers a question of Milnor (1.7).

Note that while $f$ is only $C^{n}$, the maps $\rho, \sigma$, and $\tau$ are real analytic. Simple examples (1.4) show that "topologically" cannot be replaced by " $C^{n}$ " and that no reasonable classification is possible if $p=1$. Propositions more general than (1.1) are also given ((4.7) and (4.9)).

Theorem (1.1) was announced in the talks [2] and [20]. For $n=p=2(1.1)$ was essentially proved by Stoilow [2, pp. 147 and 148] and for $n=p \geqq 3$ by Church [2, p. 155]. Both [6, p. 72, (1.5)] and [2, p. 159] deal with maps having a small singular set, and [13, §11] discusses maps with isolated critical points. The map $\tau$ is due to $\mathrm{N}$. Kuiper [13, p. 102] and it is topologically equivalent to the cone map $c(\psi)$ of the Hopf fibration $\psi: S^{3} \rightarrow S^{2}(1.10)$.

Convention 1.2. A symbol such as $M^{n}$ denotes a separable $n$ manifold, without boundary unless otherwise specified (except for obvious cases). A manifold with boundary may have empty boundary.

The boundary of a space $X$ is denoted by $X$ or $\partial X$ (in case 
$X$ is a manifold), the interior of $X$ by int $X$, and the closure of $X$ by $\bar{X}$ or $C l[X]$. The distance between two points is $d(x, y)$, and $S(x, \varepsilon)=\{y: d(x, y)<\varepsilon\}$. The space of real (resp., complex) numbers is denoted by $R$ (resp., $C$ ), euclidean $n$-space by $R^{n}$, its origin by 0 , the closed ball $C l[S(0,1)]$ in $R^{n}$ by $D^{n}$, and the sphere $\partial D$ by $S^{n-1}$.

A map is a continuous function, the restriction of a function $f$ to $X$ is denoted by $f \mid X$, and the composition of two functions by $g f$ or $g \circ f$. Homeomorphism of topological spaces and isomorphism of groups is denoted by $\approx$. The map $\pi: X \times Y \rightarrow X$ is projection, and $\iota$ is used for the identity map on a space.

Given maps $\psi: X \rightarrow Y$ and $\phi: U \rightarrow V$, define $\psi \times \phi: X \times U \rightarrow V \times Y$ by $(\dot{\psi} \times \dot{\phi})(x, u)=(\psi(x), \dot{\phi}(u))$. Define the open cone $c(X)$ as the identification space obtained from $X \times[0,1)$ by identifying $X \times\{0\}$ to a point $x^{*}$, and let the cone $\operatorname{map} c(\psi): c(X) \rightarrow c(Y)$ be the map induced by $\psi$ is $\times$ c.

Definition 1.3. If $f: M^{n} \rightarrow N^{p}$ and $g: K^{n} \rightarrow L^{p}$ are $C^{r}$ maps on $C^{r}$ manifolds $(r=0,1, \cdots)$, then $f$ and $g$ are $C^{r}$ equivalent if and only if there are $C^{r}$ diffeomorphisms $\alpha: M^{n} \rightarrow K^{n}$ and $\beta: N^{p} \rightarrow L^{p}$ such that $g \circ \alpha=\beta \circ f$. The map $f$ at $x$ is locally $C^{r}$ equivalent to $g$ at $u$ if there are open neighborhoods $U$ of $x$ and $V$ of $f(x)$ such that $f \mid U: U \rightarrow V$ is $C^{r}$ equivalent to $g$ with $\alpha(x)=u$. A $C^{\circ}$ diffeomorphism is a homeomorphism, and (locally) topologically equivalent means (locally) $C^{\circ}$ equivalent.

REMARK 1.4. Suppose that $f$ is any one of $\rho, \sigma$, or $\tau$, and let $\eta: R^{n} \rightarrow R^{n}$ be a $C^{\infty}$ homeomorphism such that $\eta$ fixes the origin, $\eta \mid\left(R^{n}-A\right)$ is a $C^{\infty}$ diffeomorphism, where $A$ is the closure of a sequence of points converging to the origin, and the rank of the Jacobian matrix of $\eta$ at points in $A$ is zero. Since the rank of $f$ at any point in $A$ away from the origin is not maximal, $f \circ \eta$ is not locally $C^{1}$ diffeomorphically equivalent at the origin to any of the maps in (1.1). Thus in the statement of (1.1) "topologically" cannot be replaced by " $C$ " ". For examples to show that no reasonable classification is possible if $p=1$ take height functions on compact manifolds which have an infinite collection of local maxima.

Definition 1.5. Given $M^{n}$ and $N^{n}$ manifolds with (possibly empty) boundary, $n \geqq p$, and a map $f: M^{n} \rightarrow N^{p}$, we now define the branch set $B_{f} \subset M^{n}$. Let $R_{+}^{m}=\left\{x \in R^{m}: x_{m} \geqq 0\right\}$, let $F=R^{n-p}$ or $R_{+}^{n-p}$, and let $G=R^{p}$ or $R_{+}^{p}$ (not respectively). Then $x \notin B_{f}$ if and only if $f$ at $x$ is locally topologically equivalent to $\pi: F \times G \rightarrow G$ at $(0,0)$. Occa- 
sionally the notation $B(f)$ is used.

In (1.1) $B_{\rho}=\phi$ while $B_{\sigma}$ and $B_{\tau}$ (and thus $B_{f}$ ) are discrete. With the definition of $B_{f}$ the Rank Theorem [2, p. 155] for $n \geqq p$ becomes:

RANK Theorem 1.6. If $f: M^{n} \rightarrow N^{p}$ is $C^{r}(r \geqq 1), n \geqq p$, and $\partial M^{n}=$ $\partial N^{p}=\phi$, then $B_{f} \subset R_{p-1}(f)$.

Question 1.7. (Milnor [13, p. 100, first problem]). Let $f: R^{n} \rightarrow R^{p}$ be a (real) polynomial map with an isolated critical point at 0 . For what dimensions $n \geqq p \geqq 2$ do nontrivial examples exist?

The topic of [13], except for $\S 11$, is certain complex polynomial maps $f: C^{n+1} \rightarrow C$ with 0 as the only critical point. These maps have a deep and very interesting structure related to exotic spheres, and their properties led Milnor to ask about real polynomial maps.

After posing this question Milnor says "It is not quite clear what 'non-trivial' should mean here. Certainly the projection.. is a trivial example". One natural definition is: $f$ is nontrivial at $x$ if and only if $x \in B_{f}$, i.e. $f$ is trivial at $x$ if and only if $f$ at $x$ is locally topologically equivalent to the projection map $\rho$.

In the complex polynomial case the study of a singularity employs a certain fibration, and analogous fibration exists in the real polynomial case. Milnor formulates his ("tentative") definition of nontrivial [13, p. 97 and p. 100] in terms of this fibration; we omit it here because it is technical. While Milnor's definition appears to be quite different from the definition we have given above, Church and Lamotke have shown [4] that they agree (at least for $n \neq 4$ ). With our formulation we can ask Milnor's question in other contexts.

Let $f: M^{n} \rightarrow N^{p}(n \geqq p \geqq 2)$ be continuous, $C^{r}(r=1,2, \cdots$; or $\infty)$, or real analytic. For what dimensions $(n, p)$ can $f$ have a nonempty discrete (or 0-dimensional) branch set $B_{f}$, and, up to local topological equivalence, what are the examples? For $n-p=0$ or 1 Theorem (1.1) answers a $C^{n}$ version of this question, and a fortiori answers Milnor's question for these dimensions. A continuous version is discussed in (4.9).

In sequels [5] the hypothesis on $\operatorname{dim} f\left(R_{p-1}(f)\right)$ is removed, and analogous results for $n-p=2$ are proved in both continuous and $C^{n}$ contexts. At first glance it seems very special to consider only the 
cases $0 \leqq n-p \leqq 2$ in these theorems, but for every $(n, p)$ with $n-p \geqq 4$ and $p \geqq 2$, Church and Lamotke constructed [4] a continuous counterexample with isolated branch point. Moreover, for $n-p=3$ and $p \geqq 6$, the nonexistence of examples depends on the Poincaré Conjecture. Thus our restriction $0 \leqq n-p \leqq 2$ in these papers is reasonable.

Definition 1.8. A map $f: X \rightarrow Y$ is proper if, for each compact set $K \subset Y, f^{-1}(K)$ is compact; $f$ is light if, for each $y \in Y$, $\operatorname{dim}\left(f^{-1}(y)\right) \leqq$ 0 ; and $f$ is monotone if each $f^{-1}(y)$ is connected. It is quasimonotone if, for each connected open set $U \subset Y$ and component $V$ of $f^{-1}(U), f(V)=U[23$, p. 151].

Theorem 1.9. (Cheeger and Kister [1, p. 151]; see also [2, p. 170]). If $f: M^{n} \rightarrow N^{n}$ is a proper map, $n \geqq p$, and $B_{f}=\phi$, then $f$ is the projection map of a fiber bundle.

(While they assume that $f$ is monotone, this hypothesis is not used in their proof. The manifolds may have nonempty boundary.)

REMARK 1.10. If $\psi: S^{3} \rightarrow S^{2}$ is the Hopf fibration, then $\tau$ in (1.1) is topologically equivalent to the cone map $c(\psi)$.

Proof. Let $S^{3}(r)$ and $S^{2}(r)$ be the spheres about $(0,0)$ of radius $r$ in $C \times C$ and $C \times R$, respectively, and let $\xi: C \times C-\{(0,0)\} \rightarrow C \times$ $R-\{(0,0)\}$ and $\zeta_{r}: S^{3}(r) \rightarrow S^{2}(r)$ be the restrictions of $\tau$. Then $\zeta_{1}$ is $\psi\left[13\right.$, p. 102, (11.6)], $\xi$ is proper, and since $R_{2}(\tau)=\{(0,0)\}, B_{\xi}=\phi$ (1.6). Thus (1.9) $\xi$ is a bundle map over $S^{2} \times(0, \infty)$, so that $\xi$ is topologically equivalent to $\zeta_{1} \times \bullet[17$, p. 53, (11.4)], and the conclusion results.

Outline of the Proof of (1.1) 1.11. For almost all the proof we work in a purely topological context, assuming topological analogs of the hypotheses of (1.1) or less. The lemmas of $\S 2$ show that for each $x \in M^{n}$ there is a manifold neighborhood $U$ of $x$ such that the restriction map $g=f \mid U, g: U \rightarrow f(U)$, is proper and $B_{g} \cap \partial U=\phi$. If $B_{g}=\phi$, then $g$ is a bundle map (1.9); thus, in general, $g$ can be viewed as a bundle map with singularities. In $\S 3$ we show that $g$ is open. In $\S 4$ we suppose that $x$ is not a point component of $g^{-1}(g(x))$ and deduce (4.5) that $g$ is a bundle map near $x$, which implies that $x \notin B_{f}$. (We use $\S 3$ and the 'almost bundle property' of $\S 2$ here.) Hence for each $x \in B_{f}, x$ is a point component of $f^{-1}(f(x))$; this is the situation of [6] and that paper yields the desired conclusion. Differentiability is used only as it is used in [6], i.e. to deduce in (1.1) that $f\left(B_{f}\right)$ is 0-dimensional 
and nicely embedded.

In several cases lemmas are stated and proved in somewhat greater generality than needed here, for use in [5] and [7].

\section{Extended embeddings.}

Definition 2.1. A map $g: J^{n-m} \times R^{m} \rightarrow L^{p-m} \times R^{m}$ is called a layer map if for each $t \in R^{m}, g\left(J^{n-m} \times\{t\}\right) \subset L^{p-m} \times\{t\}$. (In case $g$ is an embedding it is called an isotopy.) The restriction map

$$
g \mid\left(J^{n-m} \times\{t\}\right): J^{n-m} \times\{t\} \longrightarrow L^{p-m} \times\{t\}
$$

is denoted by $g_{t}$, and its branch set by $B\left(g_{t}\right)$. Frequently it is convenient to view $g_{t}$ as a map of $J^{n-m}$ into $L^{p-m}$.

Lemma 2.2. Let $\gamma_{i}: D^{p} \rightarrow$ (int $\left.D^{n-p}\right) \times D^{p}$ be disjoint embeddings with $\gamma_{i}(t)=\left(a_{i}(t), t\right)(i=1,2, \cdots, k)$. Then there is an isotopy $h: D^{n-p} \times$ $D^{p} \rightarrow D^{n-p} \times D^{p}$ such that $h$ agrees with the identity map on $\left(D^{n-p} \times\right.$ $\{0\}) \cup\left(\partial D^{n-p} \times D^{p}\right)$, and $h\left(\gamma_{i}(t)\right)=\left(a_{i}(0), t\right)$.

Proof. Let $X=\left\{a_{i}(0): i=1,2, \cdots, k\right\}$. The $\gamma_{i}$ define an isotopy

$$
\gamma: X \times D^{p} \longrightarrow\left(\text { int } D^{n-p}\right) \times D^{p}
$$

by sending $\left(a_{i}(0), t\right)$ to $\left(a_{i}(t), t\right)$, which is readily extended to a neighborhood of $X$ by sending $(u, t)$ to $\left(u-a_{i}(0)+a_{i}(t), t\right)$ for $u$ near $a_{i}(0)$. The desired ambient isotopy $h: D^{n-p} \times D^{p} \rightarrow D^{n-p} \times D^{p}$ extending $\gamma$ is given by Lees' Neighborhood $n$-Isotopy Extension Theorem [12, p. 530].

Definition 2.3. Let $f: M^{n} \rightarrow N^{p}$ be a map, let $y \in W \subset N^{p}$, and let $Q \subset f^{-1}(y)$. Define the embedding $\lambda: Q \times\{y\} \rightarrow M^{n}$ by $\lambda(q, y)=q$ for each $q \in Q$. An embedding $\nu: Q \times W \rightarrow M^{n}$ extending $\lambda$ with

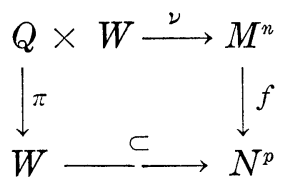

commutative is called an extended embedding of $Q$ over $W$. For each $w \in W$ let $\nu_{w}: Q \rightarrow f^{-1}(w)$ be the embedding defined by $\nu_{w}(q)=\nu(q, w)$.

An extended embedding with $Q$ a single point space is called a cross-section over $W$. (In case $f$ is a fiber bundle, it is a cross-section in the usual sense.) 
Lemma 2.4. Let $f: M^{n} \rightarrow N^{p}$ be a map, let $n \geqq p$, let $y \in N^{p}$, and let $p \subset f^{-1}(y)-B_{f}$ be a compact $(n-p)$-submanifold with bicollared boundary (e.g. let $P$ be a closed bicollar of a bicollared compact ( $n-$ $p-1)$-submanifold $\left.Q \subset f^{-1}(y)-B_{f}\right)$.

(a) Then there is an open p-cell neighborhood $D$ of $y$ and an extended embedding $\mu: P \times D \rightarrow M^{n}$ such that $B_{f} \cap$ imag $\mu=\phi$ (in the example let $\nu=\mu \mid Q \times D)$.

(b) Given $\nu$ and $Q$ as in (a) (or $Q=\phi$ ), $\delta>0$, and a family of components of $f^{-1}(y)-Q$ such that $L=\cup\{J: J \in \mathscr{J}\}$ has compact closure, $\bar{L}-L \subset Q$, and either (i) $\mathcal{J}$ is finite or (ii) each diam $J<$ $\delta / 3$, then there is a p-cell neighborhood $E$ of $y$ in $D$ such that for each $J \in \mathscr{J}$, the component $K$ of $f^{-1}(E)$-imag $\nu$ containing $J$ has (i) $d(x, J)<$ $\delta / 3$ for each $x \in K$, resp. (ii) $\operatorname{diam} K<\delta$.

Proof of (a). We will assume that the reader has read the proofs of the Theorem and the Remark in [1] and has them at hand. Let $M^{n}, N^{p}$, and $y$ be denoted by $W, Y$, and $y_{0}$, and let the $(n-p)$-manifold $P^{\prime}$ be the union of $P$ and a bicollar of its boundary. For each $x \in P$, there are closed neighborhoods $U$ of $y_{0}$ in $Y$ and $V$ of $x$ in $W$, and a homeomorphism $h: B(2) \times U \rightarrow V$ such that $f \circ h$ is the projection map onto $U$ (since $x \notin B_{f}$ ). In fact it is possible to choose the closed neighborhood $U$ and a collection of such embeddings $\left\{h_{j}: j=\right.$ $1,2, \cdots, k\}$ on $B(2) \times U$ such that $P \subset \bigcup_{j=1}^{k}$ int $h_{j}(B(1))$ and $f^{-1}\left(y_{0}\right) \cap$ $h_{j}(B(2)) \subset \operatorname{int} P^{\prime}$. For $y \in U$ define $M_{y}=f^{-1}(y)-B_{f}$ (so $P \subset P^{\prime} \subset M_{y_{0}}$ ) and $h_{y, j}: B(2) \rightarrow M_{y}$ by $h_{y, j}(t)=h_{j}(y, t)$. The proofs of [1] now yield the embedding required for (a).

Proof of (b). Let $W \subset M^{n}$ be compact with $\bar{L} \subset$ int $W$; we may suppose that $\delta<d(\bar{L}$, bdy $W)$. Let $P$ be a closed bicollar of $Q(P=$ $\phi$ if $Q=\phi$ ) and $\mu: P \times D \rightarrow M^{n}$ be as in (a); we may suppose that $D$ is sufficiently small and $P$ is a sufficiently small bicollar of $Q$ that $d(x, Q)<\delta / 3$ for each $x \in$ imag $\mu$ (remember that $Q=\mu(Q, y)$ ). Let $L^{\prime}$ be $L-\operatorname{int} P$ (since $\bar{L}-L \subset Q, L^{\prime}$ is compact). Each component $K$ of $L$ (resp. of $f^{-1}(D)$ - imag $\nu$ ) contains one and only one component $K^{\prime}$ of $L^{\prime}$ (resp., of $f^{-1}(D)$ - int imag $\mu$ ). Let $E_{r} \subset N^{p}(r=1,2, \cdots)$ be closed $p$-cells such that $E_{r+1} \subset E_{r}$ and $\bigcap_{r} E_{r}=\{y\}$. Then it suffices to prove that $\left(^{*}\right)$ there exists an integer $r$ such that, for each $J \in \mathscr{J}$, the component $H$ of $f^{-1}\left(E_{r}\right)$-int imag $\mu$ containing $J$-int $P$ has $d(x, J$-int $P)<$ $\delta / 3$ for each $x \in H$.

Suppose the contrary. Then there are components $J_{r}$ of $L^{\prime}$, components $H_{r}$ of $f^{-1}\left(E_{r}\right)$ - int imag $\mu$ with $J_{r} \subset H_{r}, y_{r} \in H_{r}$ with $d\left(J_{r}, y_{r}\right) \geqq$ $\delta / 3$, and paths $\Gamma_{r} \subset H_{r}$ joining some point $x_{r} \in J_{r}$ to $y_{r}$. We may suppose that $\Gamma_{r} \subset W$, that $x_{r} \rightarrow x_{0}$, and that $y_{r} \rightarrow y_{0}$. Thus $x_{0} \in L^{\prime}, \Gamma=$ 
$\lim \sup \Gamma_{r}$ is connected [23, p. 14, (9.1)], and $\operatorname{diam} \Gamma \geqq \delta / 3$. Now $\Gamma$ is contained in some component $J^{\prime}$ of $L^{\prime}$. In case (i) there are only a finite number of components of $L^{\prime}$, and since they are compact, all but a finite number of the $J_{r}$ are in fact $J^{\prime}$; since $d\left(J^{\prime}, y_{0}\right) \leqq \delta / 3$, a contradiction results. In case (ii), since $\operatorname{diam} J^{\prime}<\delta / 3$ and $\operatorname{diam} \Gamma \geqq$ $\delta / 3$, a contradiction results also.

Lemma 2.5. Let $f: M^{n} \rightarrow N^{n}$ be a map with $0 \leqq n-p$ and $n-$ $p \neq 4$ or 5 , let $y \in N^{p}$, let $\operatorname{dim}\left(B_{f} \cap f^{-1}(y)\right) \leqq 0$, let $X \subset B_{f} \cap f^{-1}(y)$ be compact, and let $\varepsilon>0$. Then there is a compact $(n-p-1)$-manifold $Q$ (or $\phi)$, an open p-cell neighborhood $D$ of $y$ in $N^{p}$, and an extended embedding $\nu: Q \times D \rightarrow M^{n}$ such that each component $K$ of $f^{-1}(D)$ - imag $\nu$ meeting $X$ has diam $K<\varepsilon$, their union has compact closure, and imag $\nu \cap B_{f}=\phi$.

Proof. Let $T, T^{\prime} \subset f^{-1}(y)$ be compact with $X \subset \operatorname{int} T^{\prime}, T^{\prime} \subset \operatorname{int} T$ (interior relative to $f^{-1}(y)$ ), and let $\eta>0$ be less than both $d\left(X\right.$, bdy $T^{\prime}$ ) and $d\left(T^{\prime}\right.$, bdy $\left.T\right)$. We may suppose that $\varepsilon<\eta$. Let $X^{\prime}=T^{\prime} \cap B_{f}$, and let $U_{k}=\left\{x \in T: d\left(x, X^{\prime}\right) \leqq 1 / k\right\}(k=1,2, \cdots)$.

We will first prove that (1) for $k$ sufficiently large, each component of $U_{k}$ has diameter less than $\varepsilon$. Suppose the contrary. Then there are a subsequence $\{m(k)\}$, components $\Gamma_{k}$ of $U_{m(k)}$, and points $x_{k}, y_{k} \in$ $\Gamma_{k}$ with $d\left(x_{k}, y_{k}\right) \geqq \varepsilon$. We may suppose that $x_{k} \rightarrow x_{0}$ and $y_{k} \rightarrow y_{0}$. Thus $\Gamma=\lim \sup \Gamma_{k}$ is connected [23, p. 14, (9.1)], and since $d\left(x_{0}, y_{0}\right) \geqq$ $\varepsilon$, diam $\Gamma \geqq \varepsilon$. If $k$ is fixed, then for each $j \geqq k, \Gamma_{j} \subset U_{m(j)} \subset U_{m(k)}$, so that $\Gamma \subset U_{m(k)}$. Thus $\Gamma \subset \bigcap_{k} U_{m(k)}=X$, contradicting the fact that $X$ is totally disconnected (since $X \subset B_{f} \cap f^{-1}(y)$ ). Thus (1) is true.

If a generalized continuum fails to be locally connected, it fails at (at least) a subcontinuum of points [23, p. 19, (12.3)]. Since $f^{-1}(y)-$ $B_{f}$ is an $(n-p)$-manifold (or $\left.\phi\right)$ and $B_{f} \cap f^{-1}(y)$ is totally disconnected, each component $\Lambda$ of $f^{-1}(y)$ is locally connected. Hence (2) if $U$ is open in $\Lambda$, then each component of $U$ is open in $\Lambda$.

Let $k$ be the number given by (1), and (3) let $V$ be a component of $U_{k}$ meeting $X$. Then diam $V<\varepsilon$, so that $V \subset T^{\prime}$. Let bdy $V$ refer to the boundary of $V$ in the component $\Lambda_{V}$ of $f^{-1}(y)$ containing $V$, and let $x \in \operatorname{bdy} V$. Since $V \subset U_{k}, d\left(x, X^{\prime}\right) \leqq 1 / k$; suppose $d\left(x, X^{\prime}\right)=$ $a<1 / k$. The component $W$ of $\left\{u \in \Lambda_{V}: d(x, u)<1 / k-a\right\}$ containing $x$ is open in $\Lambda_{V}$ (by (2)), $W \subset U_{k}$, and thus $W \subset \operatorname{int} V$ (relative to $\Lambda_{V}$ ), contradicting the choice of $x$; thus $(4) d(x ; X)=1 / k$ for each $x \in$ bdy $V$.

Let $\Delta$ be the closure of the union of bdy $V$ for $V$ satisfying (3) (actually the union is closed). For each $x \in \Delta, d\left(x, X^{\prime}\right)=1 / k$, and since 
$\Delta \subset T^{\prime}$ and $T^{\prime} \cap B_{f}=X^{\prime}, \Delta \cap B_{f}=\phi . \quad$ Let $L$ be the $(n-p)$-manifold int $T-B_{f}$ (possibly empty). Siebenmann and Kirby have shown (see $[15$, p. 949] that a topological manifold with dimension not 4 or 5 has a handle decomposition, so in particular there are compact $(n-p)$ manifolds with boundary $\left\{L_{j}\right\}(j=1,2, \cdots, k$ or $j=1,2, \cdots)$ such that $L_{j} \subset$ int $L_{j+1}$ and $L=\bigcup_{j} L_{j}$. Since $\Delta$ is compact, there is a $j$ such that $\Delta \subset \operatorname{int} L_{j}$. Since $\partial L_{j}$ is collared, there is a compact ( $n-$ $p$ )-manifold $P^{n}$ with boundary (or $\phi$ ) such that $\Delta \subset \operatorname{int} P^{n}, P^{n} \subset \operatorname{int} L_{j}$, (and so $P^{n} \subset L$ ), and $\partial P^{n}$ is bicollared in $L$. Let $Q=\partial P^{n}$.

Since each component $Y$ of $f^{-1}(p)-Q$ meeting $X$ is contained in some $V$ satisfying (3), $\operatorname{diam} Y<\varepsilon$. Let $\mu$ and $\nu$ be the extended embeddings given by (2.4a and $\mathrm{b})$; the conclusion results.

COROLlaRY 2.6. Let $f: M^{n} \rightarrow N^{p}$ be a map with $0 \leqq n-p$ and $n \neq 4,5$, let $x \in M^{n}$, and let $\operatorname{dim}\left(B_{f} \cap f^{-1}(f(x))\right) \leqq 0$. Then there is a connected (not neccessarily compact) manifold $K^{n} \subset M^{n}$ with boundary such that $x \in \operatorname{int} K^{n}\left(=K^{n}-\partial K^{n}\right)$, the closure $\bar{K}^{n}$ of $K^{n}$ in $M^{n}$ is compact, there is an open p-cell $D \subset N^{p}$ with $f\left(K^{n}\right) \subset D$, and the restriction map $g: K^{n} \rightarrow D$ is proper with $B_{g} \cap \partial K^{n}=\phi$.

For example, let $f: R^{2} \rightarrow R$ be projection on the first factor, let $x=(0,0)$, and let $K^{2}=(-1,1) \times[-1,1]$.

Proof. We may suppose that $x \in B_{f}$. Apply (2.5) where $X=\{x\}$, and let $K^{n}$ be the closure in $f^{-1}(D)$ of $K$. Thus $K^{n}$ is a manifold with boundary, and $\partial K^{n} \subset$ imag $\nu$ (which may be empty). Let $Y$ be a compact subset of the open 2-cell $D$. Since bdy $K^{n}$ (bdy taken relative to $\left.M^{n}\right)$ is the disjoint union of $\partial K^{n}$ and a subset of $f^{-1}(\operatorname{bdy} D)$, $g^{-1}(Y)=f^{-1}(Y) \cap K^{n}=f^{-1}(Y) \cap \bar{K}^{n}$ ) (closure in $M^{n}$ ), and so is compact; thus $g$ is proper.

\section{Open maps.}

Definition 3.1. For $f: M^{n} \rightarrow N^{p}$ and $x \in M^{n}$, let $\Gamma(x)=\Gamma_{f}(x)$ be the component of $f^{-1}(f(x))$ containing $x$. If, for every neighborhood $U$ of $x, f(x) \in \operatorname{int} f(U)$, then $f$ is open at $x$.

Lemma 3.2. Let $f: M^{n} \rightarrow N^{p}$ be a map with $n \geqq p$, and let $x \in M^{n}$ with $\operatorname{dim}\left(f^{-1}(f(x)) \cap B_{f}\right) \leqq 0$ and $\Gamma(x) \neq\{x\}$. Then $f$ is open at $x$.

Proof. Let $U$ be an open neighborhood of $x$ in $M^{n}$. For $n=p$, $f^{-1}(y)-B_{f}$ is discrete, so the hypotheses cannot be satisfied. Thus $n>p$, and there is $z \in(\Gamma(x) \cap U)-B_{f}$, so that $f(x)=f(z) \in \operatorname{int} f(U)$. 
Since $U$ is arbitrary, $f$ is open at $x$.

Lemma 3.3. Let $f: M^{n} \rightarrow N^{p}$ be a map, and let $x \in M^{n}$ with $\Gamma(x)=$ $\{x\}$. Then there is an open p-cell $D$ about $f(x)$ such that, if $K$ is the component of $f^{-1}(D)$ containing $x, f: K \rightarrow D$ is a proper map.

Proof. Use the proof of [6, p. 74, (1.14)].

Lemma 3.4. Let $f: M^{n} \rightarrow N^{p}$ be a map with $n \geqq p$ and $\operatorname{dim}\left(f^{-1}(y) \cap\right.$ $\left.B_{f}\right) \leqq 0$ for every $y \in N^{p}$, and let $E_{f}$ be the set of points at which $f$ fails to be open.

(a) Then either $E_{f}=\phi$ (so that $f$ is open) or $\operatorname{dim} f\left(E_{f}\right) \geqq p-1$.

(b) In particular, if $\operatorname{dim} f\left(B_{f}\right) \leqq p-2$, then $f$ is open.

Proof. Let $x \in E_{f}$. By (3.2) we may as well assume that (1) $\Gamma(x)=\{x\}$; let $g: K \rightarrow D$ be the proper map given by (3.3). (In case $n-p \neq 4,5$, we could use (2.6) instead.) Then $E_{g}=E_{f} \cap K$.

We observe that (2) if $V \subset K$ is open, then int $g(V) \neq \phi$, i.e. $\operatorname{dim} g(V)=p$ [11, p. 46]. If $V \not \subset B_{g}$, the conclusion is immediate. If $V \subset B_{g}$, let $U \subset V$ be a closed $n$-cell. The map $g \mid U: U \rightarrow g(U)$ is light (from the dimension hypothesis), so that [11, p. 91, VI. 7] $\operatorname{dim} g(U) \geqq$ $n$. Since $n \geqq p, \operatorname{dim} g(V)=\operatorname{dim} g(U)=n=p$.

Now suppose that $g(K) \neq D$ and $\operatorname{dim} g\left(E_{g}\right) \leqq p-2$. Since $g$ is proper, $g(K)$ is closed, so that $D-g(K)$ is a nonempty open subset of $D$. By (2) int $g(K) \neq \phi$. Thus $D-$ bdy $g(K)$ is not connected, and hence [11, p. 48] dim bdy $g(K)=p-1$. There is a $z \in K$ with $g(z) \in$ (bdy $g(K))-g\left(E_{g}\right)$, and since $g$ is open at $z, g(z) \in \operatorname{int} g(K)$, so a contradiction results. Thus (3) $\operatorname{dim} g\left(E_{g}\right) \leqq p-2$ implies $g(K)=D$.

It is immediate from (2) that (4) $\operatorname{dim} g\left(E_{g}\right) \leqq p-2$ implies $C l[K-$ $\left.g^{-1}\left(g\left(E_{g}\right)\right)\right]=K$.

From (1), (3), and (4) if $\operatorname{dim} g\left(E_{g}\right) \leqq p-2$, then $g$ satisfies the hypotheses of $[23$, p. $149,(7.81)]$ at $x$, and so $g\left(E_{g}\right)$ locally separates $D$ at $x$. A contradiction of $\left[11\right.$, p. 48] results, and hence $\operatorname{dim} g\left(E_{g}\right) \geqq$ $p-1$, yielding conclusion (a).

Since $E_{f} \subset B_{f}$, conclusion (b) follows from (a).

\section{O-regular maps.}

Definition 4.1. Let $X$ and $Y$ be metric spaces, let $f: X \rightarrow Y$ be 
open, and let $x \in X$. The map $f$ is 0 -regular at $x$ if, for every $\varepsilon>0$, there is a $\delta>0$ such that if $y \in Y$ and $u, v \in S(x, \delta) \cap f^{-1}(y)$, then there is an arc from $u$ to $v$ in $S(x, \varepsilon) \cap f^{-1}(y)$. If $f$ is proper, onto, and 0 -regular at each $x \in X$, then $f$ is 0 -regular.

Lemma 4.2. If $M^{n}$ and $N^{p}$ are manifolds with boundary, $f: M^{n} \rightarrow$ $N^{p}$ is a map, and $x \in M^{n}-B_{f}$, then $f$ is 0-regular at $x$.

The proof is immediate from (1.5).

Lemma 4.3. Let $X$ be a separable metric space, let $A \subset X$ be closed, let $X-A=M^{n}$ and $N^{p}$ be manifolds with boundary, let $n>p$, let $f: X \rightarrow N^{p}$ be proper, open, and onto, and let $B=B\left(f \mid M^{n}\right) \cup A$ (1.5). Suppose that $f(B)$ is nowhere dense, $\operatorname{dim}\left(B \cap f^{-1}(y)\right) \leqq 0$ for each $y \in N^{p}$, and $f^{-1}(z)$ is connected for each $z \in N^{p}-f(B)$.

(a) Then $f^{-1}(y)$ is path connected for each $y \in N^{p}$.

(b) If $f$ is 0 -regular, $F \approx S^{1}$ or $[0,1]$, and $f^{-1}(z) \approx F$ for each $z \in N^{p}-f(B)$, then $f$ is a bundle map with fiber $F$.

Proof. Each $y \in N^{p}$ has a compact neighborhood $V$, and $f^{-1}(V)$ is compact. There are $z_{k} \in V-f(B)$ with $z_{k} \rightarrow y$, and $f^{-1}\left(z_{k}\right) \rightarrow f^{-1}(y)$ [23, p. 10, and p. 130, (4.32)], so that $f^{-1}(y)$ is connected [23, p. 14]. Since $f^{-1}(y)$ is locally connected except possibly at most 0 -dimensional set $B \cap f^{-1}(y)$, it is locally connected [23, p.19, (12.3)], and so path connected [23, p. 38, (5.2)].

Under the hypotheses of (b) $f^{-1}\left(z_{k}\right) \rightarrow f^{-1}(y)$ 0-regularly [21, p. 482], and thus ([21, p. 484, Theorem 2] and [22, p. 341, Theorem 5.1]) $f^{-1}(y) \approx F$. By [8, p. 115, Theorem 7] $f \mid f^{-1}(V)$ is bundle map, and (b) results. This lemma is used in (4.5) and [7], and its considerable generality is required for the latter applications.

Definition 4.4. Given $f: M^{n} \rightarrow N^{p}$, the singular set $A_{f}$ (see [6]) is defined as follows: $x \in M^{n}-A_{f}$ if and only if there are open neighborhoods $U$ of $\Gamma(x)$ and $V$ of $f(x)$ such that $f \mid U: U \rightarrow V$ is topologically equivalent to the projection map $\pi: V \times \Gamma(x) \rightarrow V$. Thus (1.5) $B_{f} \subset A_{f}$. See (4.6).

LeMma 4.5. Let $f: M^{n} \rightarrow N^{p}$ be a proper map with $n \geqq p, \partial M^{n}$ possibly nonempty, $B_{f} \subset \operatorname{int} M^{n}, \operatorname{dim} f\left(B_{f}\right) \leqq p-2$, and $\operatorname{dim}\left(f^{-1}(y) \cap\right.$ $\left.B_{f}\right) \leqq 0$ for each $y \in N^{p}$.

(a) If $\Gamma(\bar{x}) \neq\{\bar{x}\}$ (see (3.1)), then there are open neighborhoods $U$ of $\Gamma(\bar{x})$ and $V$ of $f(\bar{x})$ such that $f \mid U: U \rightarrow V$ is a proper, open, monotone (onto) map. 
(b) If, in addition, $n-p \neq 4,5$, then $f \mid U$ is 0 -regular.

(c) If, in addition, $n=p+1$, then $f \mid U$ is a bundle map (thus $\left.A_{f} \cap U=\phi\right)$.

(d) If $\Gamma(x) \neq\{x\}$ for every $x \in M^{n}$ and $M^{n}$ and $N^{p}$ are connected, then $f=\psi \circ \phi$, where $\phi$ is monotone and $\psi$ is a k-to-one covering map.

Proof. By (3.4) $f$ is open. The hypotheses that $\Gamma(\bar{x}) \neq\{\bar{x}\}$ and $\operatorname{dim}\left(f^{-1}(f(\bar{x})) \cap B_{f}\right) \leqq 0$ imply that $n>p$. If $p \leqq 1$, then $B_{f}=\phi$, and the conclusions follow from $(4.2),[18$, p. $63,(2.3)]$, and $[19$, p. 661 , (2.1)]. Thus we may suppose that $n>p \geqq 2$.

Let $x \in \Gamma(\bar{x})-B_{f}$. There is a cross section $\mu$ at $x$ over an open $p$-cell $V \subset N^{p}$. Let $U$ be the component of $f^{-1}(V)$ containing $\Gamma(\bar{x})$ and let $g: U \rightarrow V$ be the restriction of $f$. Since $\operatorname{dim}\left(g^{-1}(y) \cap\left(B_{g}\right) \leqq 0\right.$, each $\operatorname{dim} g^{-1}(y) \leqq n-p$; thus by [11, p. 91, Theorem VI 7] $\operatorname{dim}\left(g^{-1}\left(g B_{g}\right)\right) \leqq$ $n-2$, so that $U^{\prime}=U-g^{-1}\left(g\left(B_{g}\right)\right)$ is path connected.

Let $\alpha: U^{\prime} \rightarrow V-g\left(B_{g}\right)$ be the restriction of $g$; since $\alpha$ is a proper map with $B_{\alpha}=\phi, \alpha$ can be factored $\alpha=\gamma_{\circ} \beta$, where $\beta$ is a monotone map and $\gamma$ is a covering map [18, p. 63, (2.3)]. Now $\beta \circ \mu \mid\left(V-g\left(B_{g}\right)\right)$ is a global cross-section of $\gamma$, so that $[16$, p. 77 (6) and (7)] $\gamma$ is a homeomorphism. Thus $\alpha$ is monotone, and by [18, p. 64, (2.5); the proof is still valid for $\partial M^{n} \neq \phi$ and $\left.B_{f} \subset \operatorname{int} M^{n}\right] g$ is monotone onto. Conclusion (a) results.

Now suppose that $\Gamma(x) \neq\{x\}$ for every $x \in M^{n}$. Since $f$ is open, and thus quasi-monotone, there is a natural number $k$ such that each $f^{-1}(y)$ has at most $k$ components, and for $y \in N^{p}-f\left(B_{f}\right), f^{-1}(y)$ has exactly $k$ components $[18$, p. $64,(2.5)]$. Let $y \in N^{p}$, and let $\Gamma_{i}(i=$ $1,2, \cdots, h \leqq k$ ) be the components of $f^{-1}(y)$. Let $U_{i}$ and $V_{i}$ be as given by (a) for $\Gamma_{i}$, and let $V$ be a $p$-cell neighborhood of $y$ such that $V \subset \bigcap_{i} V_{i}$ and the $\Gamma_{i}$ are in distinct components of $f^{-1}(V)$. Since $f$ is quasi-monotone, each component of $f^{-1}(V)$ meets some $\Gamma_{i}$, so there are exactly $h$ components $W_{i}$, where $\Gamma_{i} \subset W_{i}$. Since $f \mid W_{i}: W_{i} \rightarrow$ $V$ is monotone, for each $z \in V-f\left(B_{f}\right), f^{-1}(z)$ has $h$ components; thus $h=k$. From [18, p. 63, (2.1)] (d) follows.

For $x \in U-B_{g}, g$ is 0 -regular at $x$ (4.2). Thus, to prove (b) it suffices to prove that $g$ (or equivalently $f$ ) is 0 -regular at each $x \in B_{g}$. For $x \in B_{g}$ and $\varepsilon>0$, let $K$ be as given in (2.5) for $g$ and $X=\{x\}$, and let $\zeta: \bar{K} \rightarrow D$ be the restriction of $g$. Thus $\bar{K} \subset S(x, \varepsilon) \subset U$. Since $g$ has a global cross-section, $\Gamma_{g}(u) \neq\{u\}$ for every $u \in U$; thus $\Gamma_{\zeta}(u) \neq$ $\{u\}$, and by (d) $\zeta=\psi \circ \phi$, where $\phi$ is monotone and $\psi$ is a covering map. Since $D$ is simply connected, $\psi$ is a homeomorphism, so that $\zeta$ is monotone. 
By (4.3) (a) $\zeta^{-1}(y)$ is path connected for each $y \in D$. Choose $\delta>0$ such that $S(x, \delta) \subset \operatorname{int} K$. Since

$$
S(x, \delta) \cap f^{-1}(y) \subset \zeta^{-1}(y) \subset S(x, \varepsilon) \cap f^{-1}(y),
$$

$f$ is 0 -regular at $x$. Conclusion (b) results.

If $n=p+1$, then $g \mid\left(U-g^{-1}\left(g\left(B_{g}\right)\right)\right)$ is bundle map (1.9) with fiber a compact, connected 1 -manifold $F$, i.e., $E \approx S^{1}$ or $[0,1]$, and conclusion (c) follows from $(4.3(b))$.

Lemma 4.6. Let $f: M^{n} \rightarrow N^{p}$ be a proper map with $\Gamma(x) \subset B_{f}(3.1)$ for every $x \in B_{f}$. Then $B_{f}=A_{f}$ (4.4).

Proof. If $x \notin A_{f}$, then $x \notin B_{f}$. If $x \notin B_{f}$, then from the hypothesis $\Gamma(x) \cap B_{f}=\dot{\phi}$. There is an open $p$-cell neighborhood $V$ of $f(x)$ such that the component $U$ of $f^{-1}(V)$ containing $\Gamma(x)$ is disjoint from $B_{f}$. Since $f \mid U: U \rightarrow V$ is also proper, it is a bundle map (1.9), and since $V$ is contractible, the bundle is trivial. Thus $x \notin A_{f}$.

Proposition 4.7. Let $f: M^{n} \rightarrow N^{p}$ be $C^{n}$ with $n=p$ or $p+1, B_{f} \neq$ $\phi, \operatorname{dim} f\left(B_{f}\right) \leqq p-2$, and $\operatorname{dim}\left(f^{-1}(y) \cap B_{f} \leqq 0\right.$ for each $y \in N^{p}$. Then $\operatorname{dim} B_{f}=2 p-n-2$ and there is a closed subset $X \subset B_{f}$ such that $\operatorname{dim} X<2 p-n-2$ and, for each $x \in B_{f}-X, f$ at $x$ is locally topologically equivalent to the layer map

$$
c(\psi) \times \iota: D^{2(n-p+1)} \times R^{2 p-n-2} \rightarrow D^{n-p+2} \times R^{2 p-n-2}
$$

(see (1.2)), where $c(\psi)=\sigma$ (see (1.1)) if $n=p$, and $c(\psi)=\tau$, i.e. (1.10) the cone map of the Hopf fibration $\psi: S^{3} \rightarrow S^{2}$, if $n=p+1$.

Proof. Let $X$ be the set of all $x \in B_{f}$ such that $f$ at $x$ is not locally equivalent to $c(\psi)$; then $X$ is closed.

Let $x \in B_{f}$, and let $K$ be the neighborhood of $x$ and $g: K \rightarrow D$ be the proper map given by (2.6). If $n=p$, then for each $y \in D, g^{-1}(y)-$ $B_{f}$ is discrete and $\operatorname{dim}\left(g^{-1}(y) \cap B_{f}\right) \leqq 0$, so that $g$ is light, i.e. each $\Gamma(u)=\{u\}$. If $n=p+1$ it follows from (4.5(c)) that for each $u \in B_{g}$, $\Gamma(u)=\{u\}$. There is an open $p$-cell neighborhood $U \subset D$ of $f(x)$ sufficiently small that the component $V$ of $f^{-1}(U)$ containing $x$ has $\bar{V} \subset \operatorname{int} K$. Since $\bar{K}$ is compact, the restriction map $h: V \rightarrow U$ is proper, so that (4.6) $B_{h}=A_{h}$.

Since $\operatorname{dim} f\left(B_{f}\right) \leqq p-2$ and $B_{f} \neq \phi, p \geqq 2$. In case $n=p, h$ is light, and since $\operatorname{dim} h\left(B_{h}\right) \leqq p-2$, the Jacobian determinant of $h$ is (locally) nonnegative or nonpositive [3, p. 94, (2.3) and p. 98, (1.7)]. 
In both cases by $\left[6\right.$, p. 83, (4.1)] there is a closed set $Y_{h} \subset h\left(A_{h}\right)$ such that $\operatorname{dim} Y_{h}<\operatorname{dim} h\left(A_{h}\right)$ and, for each $x \in A_{h}-h^{-1}\left(Y_{h}\right), h$ at $x$ is locally topologically equivalent to $c(\psi) \times \iota$. Thus $\operatorname{dim} h\left(A_{h}\right)=2 p-$ $n-2$ and $\operatorname{dim} A_{h} \geqq 2 p-n-2$. Since $h \mid A_{h}$ is $\operatorname{light,} \operatorname{dim} A_{h} \leqq$ $\operatorname{dim} h\left(A_{h}\right)$ [11, p. 91, Theorem VI 7], so that $\operatorname{dim} A_{h}=2 p-n-2$, and $\operatorname{dim}\left(A_{h} \subset h^{-1}\left(Y_{h}\right)\right) \leqq \operatorname{dim} Y_{h} \leqq 2 p-n-2$. Since $A_{h}=B_{h}$ and $V \cap$ $X \subset A_{h} \cap h^{-1}\left(Y_{h}\right), \operatorname{dim}(V \cap X)<2 p-n-2$; since $x \in B_{f}$ was arbitrary and $V$ is a neighborhood of $x, \operatorname{dim} X<2 p-n-2$.

REMARK 4.8. Theorem (1.1) is a Corollary of (4.7). (Use the Rank Theorem (1.6).)

The next result (4.9) is a topological analog of (1.1). If $f: M^{p+1} \rightarrow$ $N^{p}$ is continuous with $p \geqq 2$ and $B_{f}$ discrete, then $f$ satisfies the hypotheses of (4.9); in this case the result was proved by Timourian [19].

Proposition 4.9. Let $f: M^{n} \rightarrow N^{p}$ be a map with $n=p$ or $p+1$ and $p \geqq 2$, let $\operatorname{dim} B_{f} \leqq 0$, and let $\operatorname{dim} f\left(B_{f}\right) \leqq 0$. If $p \geqq 3$ suppose in addition that for each $y \in f\left(B_{f}\right)$ and neighborhood $W$ of $y$, there is an open p-cell $U$ such that $y \in U \subset W$ and $U-f\left(B_{f}\right)$ is simply connected. Then at each $x \in M^{n}, f$ is locally topologically equivalent to one of the maps $\rho, \sigma$, or $\tau$ of (1.1).

Proof. By the first two paragraphs of the proof of (4.7), for each $x \in B_{f}$ there is a neighborhood $V$ of $x$ such that the restriction $h: V \rightarrow$ $U$ of $f$ is proper and $B_{h}=A_{h}$. By [6, p. 75, (2.3)] at each $u \in B_{h}, h$ at $u$ is locally topologically equivalent to $\sigma$ or to $c(\psi)$, where $\psi$ is the Hopf map, i.e. to $\sigma$ or $\tau$ by (1.10).

Proposition 4.10. Let $f: J^{n-m} \times R^{m} \rightarrow L^{p-m} \times R^{m}$ be a $C^{n-p+1}$ layer map with $n-p=0,1$, or 2 , and $\operatorname{dim}\left(B_{f} \cap f^{-1}(y, t)\right) \leqq 0$ for each $t \in R^{m}$. Then $B_{f}=C l\left[\bigcup_{t}\left\{B\left(f_{t}\right)\right\}: t \in R^{m}\right]$.

By Sard's Theorem (e.g. [2, p. 156]) $\operatorname{dim}\left(f_{t}\left(R_{p-m-1}(f(t))\right) \leqq p-\right.$ $m-1$, and by the Rank Theorem (1.6) $\left(L^{p-m} \times\{t\}\right)-f\left(B_{f}\right)$ is dense in $L^{p-m} \times\{t\}$ for each $t \in R^{p-m}$. Our proof uses only this last statement, rather than $C^{n-p+1}$.

Proof. If $(x, s) \notin B_{f}$, then there is a layer embedding $\lambda:\left(D^{n-p} \times\right.$ $\left.D^{p-m}\right) \times D^{m} \rightarrow J^{n-m} \times R^{m}$ witn $(x, s) \in \operatorname{int}$ imag $\lambda, D^{p-m} \subset L^{p-m}, \pi: D^{n-p} \times$ $\left(D^{p-m} \times D^{m}\right) \rightarrow L^{p-m} \times R^{m}$ projection, and $f \circ \lambda=\pi$. For each $(v, t) \in$ int imag $\lambda, \lambda_{t}: D^{n-p} \times D^{p-m} \rightarrow J^{n-m}$ is an embedding with $v \in$ int imag $\lambda_{t}$ and $f_{t} \circ \lambda_{t}=\pi_{t}$. Thus $(v, t) \notin B\left(f_{t}\right)$, so that $(x, s) \notin C l\left[\bigcup_{t} B\left(f_{t}\right)\right]$. 
Suppose that $(x, s) \notin C l\left[\bigcup_{t} B\left(f_{t}\right)\right]$, but $(x, s) \in B_{f}$. Choose $\eta>0$ such that $S((x, s), \eta) \cap C l\left[\bigcup_{t} B\left(f_{t}\right)\right]=\phi$, and let $K$ be the set given by (2.5) for $f, X=\{(x, s)\}$, and $\varepsilon=\eta$, over (we may suppose) $D=U \times$ $V$, where $U$ and $V$ are open $(p-m)$ - and $m$-cells, respectively. Let $\bar{K}$ be the closure of $K$ in $f^{-1}(U \times V)$, and let $f: \bar{K} \rightarrow U \times V$ and $g_{t}: \bar{K} \cap$ $\left(J^{n-m} \times\{t\}\right) \rightarrow U \times\{t\}$ be restrictions of $f$. Each is a proper map. Since $B\left(g_{t}\right)=\phi$, each $g_{t}$ is a bundle map (1.9): call its fiber $F_{t}$.

For $u \in V$ there exists $y$ with $(y, u) \in(U \times\{u\})-g\left(B_{g}\right)$. Choose open $k$-cell $\Gamma$ and $m$-cell $\Delta$ neighborhoods of $y$ and $u$, respectively, such that $(\Gamma \times \Delta) \cap g\left(B_{g}\right)=\phi$. Since $g \mid g^{-1}(\Gamma \times \Delta)$ is a bundle map (1.9), $F_{t}$ is independent of $t$ for $t \in \Delta$. Since $u$ is arbitrary and $V$ is connected, $F_{t}$ is independent of $t$ for $t \in V$, i.e., $g^{-1}(y, t)$ is independent of $y$ and $t$.

By (3.2) $g$ is open, and thus [23, p. 152, (8.1) and (8.11)] quasimonotone. Since the number of components of $g^{-1}(y, t)$ is independent of $y$ and $t, g=\psi \circ \phi$, where $\phi$ is monotone and $\psi$ is a covering map $[18$, p. $63,(2.1)]$. Since $U \times V$ is simply connected, $\psi$ is a homeomorphism, so that $g$ is monotone, i.e., $F_{t}$ is connected. In case $n-p=$ 0 each $g_{t}$ is a homeomorphism, so that the open map $g$ is also one-toone and onto-thus $g$ is a homeomorphism, contradicting the choice of $(x, s)$. In case $n-p=1$ or 2 to obtain a contradiction it suffices [10, p. 527, Theorem B and p. 530, Corollary 2] to prove that $g$ is 0 -regular.

Given $(z, u) \in B_{g}$ and $\varepsilon>0$, let $T \subset g^{-1}(g(z, u))-\partial K$ be a closed $(n-p)$-cell with $(z, u) \in$ int $T, \partial T \cap B_{g}=\phi$, and $\operatorname{dim} T<\varepsilon$. Let $M^{n}=$ $K, Q=\partial T$, and let $\nu: Q \times D \rightarrow K$ be the extended embedding given by $(2.4(\mathrm{a}))$; we may suppose $(2.4(\mathrm{~b}))$ that the component $X$ of $g^{-1}(D)-$ imag $\nu$ containing int $T$ has $\operatorname{dim} X<\varepsilon$ and $X \cap \partial K=\phi$. Since each $g^{-1}(y, t)$ is a compact connected $(n-p)$ - manifold with nonempty boundary $(n-p=1$ or 2$)$ and $\nu(Q \times\{t\}) \approx \partial T \approx S^{n-p-1}$, it follows from the cohomology sequence with compact supports of this pair that $g^{-1}(y, t)-\nu(Q \times\{t\})$ has either one or two components. Since $g^{-1}(y, t) \cap$ $X$ contains any component it meets, there are two and $g^{-1}(y, t) \cap X$ is one of them. Choose $\delta>0$ such that $S((z, u), \delta) \subset X$. Then for every $(y, t) \in U \times V$,

$$
g^{-1}(y, t) \cap S((z, u), \delta) \subset g^{-1}(y, t) \cap X \subset g^{-1}(y, t) \cap S((z, u), \varepsilon),
$$

so that $g$ is 0 -regular at $(z, u)$. Since $g$ is 0 -regular at each $X \notin B_{f}$ (4.2), $g$ is 0 -regular.

While (4.10) is not used in this paper, [7] refers to (4.10) and (4.11), they will be used elsewhere, and the proof of (4.10) fits natu- 
rally into this section. For these reasons, they are given here.

REMARK 4.11. The hypothesis $\operatorname{dim}\left(B_{f} \cap f^{-1}(y, t)\right) \leqq 0$ is (surprisingly) essential. There is a proper layer map $f:([-2,2] \times R) \times R \rightarrow$ $R \times R$ such that each $f_{t}$ is topologically equivalent to the projection map, $f_{0}$ is the projection map, and $B_{f}=([-1,1] \times R) \times\{0\}$. For example each $f^{-1}(0, t)(t \neq 0)$ might be the union of the three subsets of $([-2,2] \times R) \times\{t\}$ defined by:

(1) $x=\sin t^{-1} y$ for $|y| \leqq 3 \pi t / 2$, (2) $y=3 \pi t / 2$ for $-2 \leqq x \leqq-1$ and (3) $y=-3 \pi t / 2$ for $1 \leqq x \leqq 2$. Open maps similar to this have been defined in [14, p. 9] and [9, p. 341].

\section{REFERENCES}

1. J. Cheeger and J. M. Kister, Counting topological manifolds, Topology, 9 (1970), 149-151.

2. P. T. Church, Differentiable monotone mappings and open mappings, pp. 145-183 in The Proceedings of the First Conference on Monotone Mappings and Open Mappings, edited by L. F. McAuley, Oct. 8-11, 1970, SUNY at Binghamton, Binghamton, N. Y., 1971.

3. — Differentiable open maps on manifolds, Trans. Amer. Math. Soc., 109 (1963), 87-100.

4. P. T. Church and K. Lamotke, in preparation.

5. P. T. Church, and J. G. Timourian, Differentiable maps with 0-dimensional critical set, II and III (mimeographed).

6. P. T. Church and J. G. Timourian, Fiber bundles with singularities, J. Math. Mech., 18 (1968), 71-90.

7. P. T. Church, and J. G. Timourian, Real analytic open maps (mimeographed).

8. E. Dyer and M. E. Hamstron, Completely regular mappings, Fund. Math., 45 (1957), 103-118.

9. W. C. Fox, The critical points of peano interior functions defined on 2-manifolds, Trans. Amer. Math. Soc., 83 (1956), 338-370.

10. M. E. Hamstrom and E. Dyer, Regular mappings and the space of homeomorphisms on a 2-manifold, Duke Math. J., 25 (1958), 521-532.

11. W. Hurewicz and H. Wallman, Dimension Theory, 2nd edition, Princeton University Press, Princeton 1948.

12. J. A. Lees, Immersions and surgeries of topological manifolds, Bull. Amer. Math. Soc., 75 (1969), 529-534.

13. J. Milnor, Singular Points of Complex Hypersurfaces, Annals of Math. Studies No. 61, Princeton Univ. Press, Princeton, N. J. 1968.

14. W. D. Nathan, Open Mappings on Manifolds, Ph. D. dissertation, Syracuse University, Syracuse, 1968.

15. R. Schultz, Some recent results on topological manifolds, Amer. Math. Monthly, 78 (1971), 941-952.

16. E. H. Spanier, Algebraic Topology, McGraw-Hill, New York 1966.

17. N. Steenrod, The Topology of Fiber Bundles, Princeton University Press, Princeton 1951.

18. J. G. Timourian, Fiber bundles with discrete singular set, J. Math. Mech., 8 (1968), $61-70$.

19. Maps with discrete branch sets between manifolds of codimension one, Canad. J. Math., 23 (1969), 660-668. 
20. J. G. Timourian, Maps with 0-dimensional critical set, in The Proceedings of Liverpool Singularities Symposium I, edited by C. T. C. Wall, Lecture Notes in Mathematics 192, Springer-Verlag, Berlin, 1971.

21. P. A. White, Regular convergence of manifolds with boundary, Proc. Amer. Math. Soc., 4 (1953), 482-485.

22. - Some characterizations of generalized manifolds with boundary, Canad. J. Math., 4 (1954), 329-342.

23. G. T. Whyburn, Analytic Topology, 2nd edition, Amer. Math., Society, Providence 1963.

Received March 13, 1970 and in revised form January 17, 1972 . Work of the first author supported by NSF Grant GP-6871; that of the second author by NSF Grant GP8888 and NRC Grant \# A7357.

SyRACUSE UNIVERSITY, UNIVERSity OF TENNESSEE, AND

UNIVERSity OF Alberta 


\title{
PACIFIC JOURNAL OF MATHEMATICS
}

\author{
EDITORS
}

H. SAMELSON

Stanford University

Stanford, California 94305

C. R. HOBBY

University of Washington

Seattle, Washington 98105

\section{J. DugundJI}

Department of Mathematics

University of Southern California

Los Angeles, California 90007

RICHARD ARENS

University of California

Los Angeles, California 90024

\section{ASSOCIATE EDITORS}
E. F. BeCKeNBACH
B. H. NeUmanN
F. WoLF
K. YoSHIDA

\section{SUPPORTING INSTITUTIONS}

\author{
UNIVERSITY OF BRITISH COLUMBIA \\ CALIFORNIA INSTITUTE OF TECHNOLOGY \\ UNIVERSITY OF CALIFORNIA \\ MONTANA STATE UNIVERSITY \\ UNIVERSITY OF NEVADA \\ NEW MEXICO STATE UNIVERSITY \\ OREGON STATE UNIVERSITY \\ UNIVERSITY OF OREGON \\ OSAKA UNIVERSITY
}

\author{
UNIVERSITY OF SOUTHERN CALIFORNIA \\ STANFORD UNIVERSITY \\ UNIVERSITY OF TOKYO \\ UNIVERSITY OF UTAH \\ WASHINGTON STATE UNIVERSITY \\ UNIVERSITY OF WASHINGTON

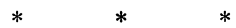 \\ AMERICAN MATHEMATICAL SOCIETY \\ NAVAL WEAPONS CENTER
}

The Supporting Institutions listed above contribute to the cost of publication of this Journal, but they are not owners or publishers and have no responsibility for its content or policies.

Mathematical papers intended for publication in the Pacific Journal of Mathematics should be in typed form or offset-reproduced, (not dittoed), double spaced with large margins. Underline Greek letters in red, German in green, and script in blue. The first paragraph or two must be capable of being used separately as a synopsis of the entire paper. The editorial "we" must not be used in the synopsis, and items of the bibliography should not be cited there unless absolutely necessary, in which case they must be identified by author and Journal, rather than by item number. Manuscripts, in duplicate if possible, may be sent to any one of the four editors. Please classify according to the scheme of Math. Rev. Index to Vol. 39. All other communications to the editors should be addressed to the managing editor, Richard Arens, University of California, Los Angeles, California, 90024.

50 reprints are provided free for each article; additional copies may be obtained at cost in multiples of 50 .

The Pacific Journal of Mathematics is published monthly. Effective with Volume 16 the price per volume (3 numbers) is $\$ 8.00$; single issues, $\$ 3.00$. Special price for current issues to individual faculty members of supporting institutions and to individual members of the American Mathematical Society: $\$ 4.00$ per volume; single issues $\$ 1.50$. Back numbers are available.

Subscriptions, orders for back numbers, and changes of address should be sent to Pacific Journal of Mathematics, 103 Highland Boulevard, Berkeley, California, 94708.

PUBLISHED BY PACIFIC JOURNAL OF MATHEMATICS, A NON-PROFIT CORPORATION

Printed at Kokusai Bunken Insatsusha (International Academic Printing Co., Ltd.), 270, 3-chome Totsuka-cho, Shinjuku-ku, Tokyo 160, Japan. 


\section{Pacific Journal of Mathematics}

\section{Vol. 41, No. $3 \quad$ BadMonth, 1972}

George E. Andrews, Two theorems of Gauss and allied identities proved arithmetically.................................. 563

Stefan Bergman, On pseudo-conformal mappings of circular domains . . . . 579

Beverly L. Brechner, On the non-monotony of dimension ............ 587

Richard Anthony Brualdi and John H. Mason, Transversal matroids and Hall's theorem .................................... 601

Philip Throop Church and James Timourian, Differentiable maps with

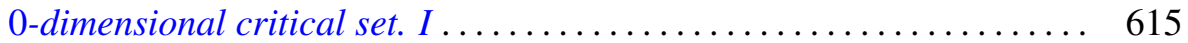

John H. E. Cohn, Squares in some recurrent sequences ............. 631

Robert S. Cunningham, Edgar Andrews Rutter and Darrell R. Turnidge, Rings of quotients of endomorphism rings of projective modules ......

Eldon Dyer and S. Eilenberg, An adjunction theorem for locally equiconnected spaces................................... 669

Michael W. Evans, On commutative P. P. rings................. 687

Ronald Lewis Graham, Hans Sylvain Witsenhausen and Hans Zassenhaus, On tightest packings in the Minkowski plane ..................

Stanley P. Gudder, Partial algebraic structures associated with

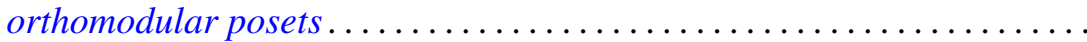

Karl Edwin Gustafson and Gunter Lumer, Multiplicative perturbation of

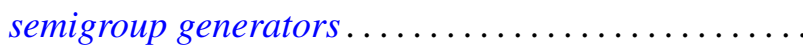

Kurt Kreith and Curtis Clyde Travis, Jr., Oscillation criteria for selfadjoint elliptic equations...

Lawrence Louis Larmore, Twisted cohomology theories and the single obstruction to lifting...........................

Jorge Martinez, Tensor products of partially ordered groups . . .

Robert Alan Morris, The inflation-restriction theorem for Amitsur cohomology ...

Leo Sario and Cecilia Wang, The class of $(p, q)$-biharmonic functions ...

Manda Butchi Suryanarayana, On multidimensional integral equations of

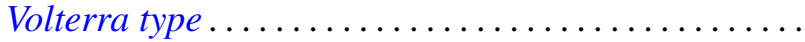

